\title{
Sintomatología vasomotora y terapias alternativas a la terapia hormonal de reemplazo
}

\author{
Camil Castelo-Branco; Alex Sanjuán; Juan José Vicente; Juan E. Blümel*
}

\section{RESUMEN}

Los sofocos son el síntoma más frecuente y característico del climaterio. Se desencadenan con el estrés, altas temperaturas ambientales y alcohol. La etiología es la deprivación estrogénica, pero los mecanismos exactos que los producen se desconocen, pero se postula que se relacionan con un cambio en la vía noradrenérgica que modifica tanto la regulación de los centros hipotalámicos, que controlan la temperatura corporal, como las neuronas productoras de GnRH. Los sofocos hay que diferenciarlos de cuadros clínicos similares como el hipertiroidismo, carcinoide, feocromocitoma; y, de reacciones a fármacos como nifedipina, niacina o calcitonina. El tratamiento más efectivo son los estrógenos. Cuando hay contraindicaciones o rechazo a esta terapia pueden plantearse otras alternativas como tibolona, gestágenos y andrógenos; entre las alternativas no hormonales figuran veralipride, alfametildopa, clonidina, metoclorporpamida y la beta-alanina. Otra medida con cierta efectividad es el aumento de la actividad física. Las mujeres japonesas presentan menos bochornos que las occidentales, relacionándose este hecho a los fitoestrógenos que ingicren a través de la soya. Diferentes fitoestrógenos se han usado con éxito en el control de los bochornos. Conclusión: cuando no se puede o no se quiere usar estrógenos existen otras alternativas terapéutas efectivas.

PALABRAS CLAVES: Menopausia, climaterio, sofocos, bochornos, tratamiento del climaterio.

\section{SUMMARY}

Hot Flashes are the most frequent symptom and characteristic of the climacteric. They are unchained with the stress, high environmental temperatures and alcohol. The etiology is the estrogenic deprivation, but the exact mechanisms that produce them are ignored, but it are postulated that they are related with a change in the noradrenergic via that modifies the regulation of the hypothalamic centers, that controls the corporal temperature, so much like the neurons producing GnRH. The hotflashes is necessary to differentiate those of clinical similar diseases like the hyperthyroidism, carcinoide, pheochromocitomas; and, of reactions to drugs like niphedipin, niacinamide or calcitonine. The most effective treatment is the estrogens. When there are contraindications or rejection to this therapy could think about other alternatives like tibolone, gestagens and androgens; between the alternatives non hormonals figures veralipride, alphamethyldopa, clonidina, metoclorpropamide and beta-alanine. Another measure with certain effectiveness is the increase of the physical activity. The Japanese women present fewer hot flashes than the Westerners, being related this fact to phytoestrogens that they ingest through the soy. Several phytoestrogens has been used with success in the control of hot flashes. Conclusion: When one is not able to or is not wanted to use estrogens exist other therapeutic effective alternatives.

KEY WORDS: Menopause, climacteric, hot flashes, hormone replacement therapy.

\section{Introducción}

Como las hormonas ováricas actúan no sólo a nivel de los órganos genitales, sino que también en el resto del organismo, el déficit de estrógenos que acontece en la menopausia va a tener efectos múltiples. A nivel del sistema nervioso central, este déficit puede manifestarse por sofocos, cefaleas, trastornos del sueño y del ánimo. afectando fuertemente la calidad de vida y contrapesando el beneficio que significa para algunas mujeres el cese de las menstruaciones y de la fertilidad (1)

Correspondence address: Camil Castelo-Branco. Dept. Gynecol. \& Obstetrics. Hospital Clínic i Provincial (Barcelona). C/villarroel 170. 08036 barcelona (spain)

Dept. Ginecología i Obstetricia. Hospital Clinic Universitari Barcelona. Facultat de Medicina. Universitat de Barcelona. Hospital Barros Luco-Trudeau. Facultad de Medicina. Universidad de Chile.
Los sofocos son el síntoma más típico y frecuente del síndrome climatérico (2), tanto es así que para ciertos autores es el único síntoma verdaderamente propio del climaterio (3) y para muchas mujeres es la referencia con la que marcan el fin de su vida reproductiva, aún cuando menstrúen (4). Estos trastornos vegetativos se acompañan de una constelación de síntomas como sudor nocturno, parestesias, palpitaciones, taquicardia, opresión precordial, mareos (5); y, también de síntomas psíquicos como irritabilidad, ansiedad y depresión (6).

En un estudio (7) se valoró la respuesta vasoconstrictora digital a la aplicación de hielo, en mujeres con y sin bochornos, observándose que en el 90\% de las mujeres con sofocos no se producía la vasoconstricción que normalmente provoca este test, sugiriendo la existencia de una anomalía de funcionamiento del sistema nervioso autónomo.

La incidencia de los bochornos varía según las estadísticas. Bates los encuentra en un 75 a $85 \%$ de las 
mujeres climatéricas (8). Parrilla y Abad en una revisión de 1987 las sitúan en cambio entre el 30\% y el 35\% (9). En Barcelona (datos no publicados), un $82 \%$ de las pacientes que consultan en nuestra unidad de menopausia manifestaban presentar bochornos.

Los bochornos aparecen tanto en las mujeres con menopausia espontánea como en las que han sido castradas (8), siendo la frecuencia de aparición de las mismas muy variable, desde presentar pocos sofocos a la semana hasta más de 15 al día (10). Pueden aparecer meses o hasta incluso años antes de la menopausia y también se pueden prolongar años después de la misma. Blümel (11) en Chile, en una población general de mujeres de 40 a 59 años de edad, los encuentra en $62 \%$ de las perimenopáusicas, persistiendo los sofocos en $35 \%$ de la mujeres con más de 5 años de menopausia.

Los síntomas vasomotores son la más notoria manifestación del inicio del climaterio, así como los más característicos (5). Se distinguen entre ellos los sofocos en forma de vasodilatación cutánea, la sudoración nocturna y el aumento de la frecuencia cardíaca en forma de palpitaciones.

El número e intensidad varían mucho de paciente a paciente, así algunas mujeres lo soportan sin tratamiento mientras que para otras puede ser una verdadera limitación socioprofesional que afecta en gran medida su calidad de vida.

Son los síntomas más frecuentes de la menopausia. Así pueden afectar hasta más del $75 \%$ de las mujeres de raza caucasiana (8), pero existen amplias variaciones según la raza; por ejemplo, en Japón sólo un $20 \%$ de las mujeres se ven afectadas. Otras razas como la indonesia o la maya también se ven menos afectadas.

Empiezan generalmente unos dos años antes de la menopausia y van disminuyendo de forma gradual la intensidad y frecuencia. Hasta un $20 \%$ de las mujeres los siguen sufriendo hasta 5 - 10 años después de la menopausia en la raza caucasiana. E incluso algunas mujeres los refieren hasta los setenta años o más.

Clásicamente se dividen en 3 fases. La primera o pródromos en la cual la paciente se despierta por la noche antes de que aparezcan los síntomas objetivos, como con una ráfaga de calor unos 45 segundos antes a continuación viene una segunda fase caracteriž́ada por una sensación de calor localizada en cara y cuello inicialmente que se va extendiendo a tórax y hombros pudiendo generalizarse a todo el cuerpo. Esto se objetiva con una vasodilatación periférica con enrojecimiento de las zonas y aumento de temperatura, asociado además a sudoración y taquicardia. Típicamente la paciente tratará de enfriarse abriendo ventanas o disminuyendo la calefacción. Finalmente se entra en la fase de resolución con temblores y escalofríos con posible sensación de frescor. Todo el proceso de la bochorno junto puede durar desde unos segundos hasta varios minutos, con una duración media de 4 minutos. Pueden aparecer desde varias veces en una hora hasta 1-2 veces al mes limitando más o menos la vida de la paciente según la frecuencia.

Las mujeres afectadas pueden experimentar todos los síntomas o sólo una parte de ellos. Pueden haber situaciones desencadenantes de los mismos como una temperatura ambiental alta, comidas o bebidas calientes, una ropa cálida, consumo de alcohol, estrés emocional o aparecer sin ninguna causa previa. Asimismo en ambientes fríos son menos frecuentes que en ambientes calurosos.

\section{Fisiopatología}

Pese a ser los síntomas más frecuentes de la menopausia los mecanismos fisiológicos que los desencadenan no están del todo claros y siguen siendo tema de controversia. Existen distintas hipótesis difíciles todas ellas de demostrar para explicar los síntomas vasomotores. Así kronenberg (12) señala que durante los pródromos existe un aumento de adrenalina que puede dar lugar a palpitaciones, seguido después por una disminución de noradrenalina a nivel periférico que da lugar a la vasodilatación y al aumento de flujo sanguíneo cutáneo. Esta vasodilatación es la que da el aumento de temperatura y ésta a su vez la transpiración y sudoración. La sudoración desembocará en una pérdida de calor, dando la fase de resolución con frescor.

Algunos autores sostienen que los sofocos serían una activación de la pérdida de calor determinada a nivel central, pero sin haber aumentado la temperatura corporal de forma real. Esto vendría determinado por un descenso del punto de regulación (set point) de la temperatura a nivel del termostato hipotálamico (13-14), el cual perdería el control como consecuencia del déficit estrogénico. La vasodilatación y sudoración posterior sería el intento corporal de alcanzar la nueva temperatura determinada por el termostato central para restaurar el equilibro. El hecho de que los síntomas vasomotores comiencen con la menopausia obligan a vincular a las hormonas sexuales con la etiopatogenia de los bochornos. El aumento de LH como de GnRH que existe en la menopausia, en cambio, no parecen estar involucrados, ya que su freno no elimina los sofocos.

Parece ser que serían las complejas relaciones que existen a nivel central entre las hormonas sexuales y distintos neurotransmisores como la noradrenalina, dopamina, serotonina y opiáceos endógenos, lo que explicaría estos síntomas. Durante la etapa reproductiva los estrógenos frenarían las vías noradrenérgicas centrales. Al llegar la menopausia las vías noradrenérgicas dejarían de estar frenadas, hiperactivándose y causando un descenso del punto de ajuste de la termorregulación, lo cual desencadenaría los sofocos. Freedman (14) recientemente parece ser que apoyaría esta hipótesis de activación central de noradrenalina.

Distintos péptidos se han vinculado con la etiopatogenia de los sofocos. Existe, por ejemplo, un aumento de péptidos relacionados con la calcitonina que son potentes vasodilatadores (15). A mayor concentración más sofocos habrá, alcanzando el nivel máximo en la máxima intensidad del sofoco. Asimismo encontramos estudios que muestran variaciones de las endorfinas durante los sofocos (15-17). Hay similitudes entre el síndrome de deprivación de opiáceos (sofocos, enrojecimiento de la cara y tronco, transpiración, temblores y trastornos del sueño) y la carencia estrogénica (17). No obstante, ciertas manifestaciones de la privación de opiáceos no se observan durante la menopausia. Los 
estrógenos estabilizan el centro hipotalámico regulador de la temperatura al mantener la actividad opioide a dicho nivel y su deprivación causa asimismo una deprivación de opioides en el hipotálamo, llevando a la inestabilidad del centro termorregulador (18).

Otros muestran además de aumentos de noradrenalina, aumentos de ácidos grasos libres, sugiriendo una activación del sistema simpático, que llevaría a cabo un aumento de la lipólisis (19). Esta lipólisis, sugieren, podría estar relacionada con una hipertrigliceridemia que tendrían especialmente aquellas mujeres que presentasen mayores y más severos síntomas climatéricos.

Todas estas hipótesis, de todas formas, no se han podido probar claramente en forma experimental, ya que no es correcto hacerlo en humanos; en animales no se ha encontrado el modelo experimental adecuado. Lo que sí está claro es que el desencadenante de los síntomas es el descenso de los estrógenos y no así el déficit o ausencia de los mismos, ya que sólo se presentan bochornos cuando ha habido una exposición previa a los mismos; serían un síndrome de privación de estrógenos. Así, el cambio hormonal que se produce al pasar de la fase fértil a la postmenopáusica, provocaría cambios de los neurotransmisores a nivel central que explicarían dichos síntomas.

\section{Diagnóstico diferencial}

Puede existir en determinadas ocasiones la posibilidad de tener que realizar un diagnóstico diferencial entre los bochornos climatéricos y otras sintomatologías parecidas (20-21). El contexto clínico junto con una cuidada exploración física y escasas pruebas complementarias, nos permitirán establecer sin dificultad un diagnóstico en la mayoría de ocasiones. En la tabla 1 se detallan las posibilidades de diagnóstico diferencial.

Tabla 1

\section{CAUSAS MAS COMUNES DE BOCHORNOS: DIAGNOSTICO DIFERENCIAL CON LOS BOCHORNOS PROPIOS DE LA MENOPAUSIA}

\begin{tabular}{|ll|}
\hline Causas endocrinas & $\begin{array}{l}\text { Hipertiroidismo } \\
\text { Síndrome carcinoide } \\
\text { Feocromocitoma } \\
\text { Reacción insulínica a la } \\
\text { diabetes }\end{array}$ \\
\hline Causas neurológicas & $\begin{array}{l}\text { Epilepsia diencefálica } \\
\text { Ansiedad }\end{array}$ \\
\hline Fármacos & $\begin{array}{l}\text { Nitroglicerina, nifedipina, } \\
\text { niacina, vancomicina, calcito- } \\
\text { nina, etanol, glutamatosódico, } \\
\text { disulfiram, hormonaliberado- } \\
\text { ra de la corticotropina }\end{array}$ \\
\hline
\end{tabular}

Deprivación del

síndrome alcohólico

\section{Tratamiento}

Este es el campo donde se ha realizado una mayor cantidad de ensayos terapéuticos alternativos a la terapia de reemplazo hormonal con diferentes grupos de substancias (Tablas 2, 3). Para el tratamiento de los mismos, cuando la administración de estrógenos no está indicada, se han propuesto fármacos como el naproxeno, la clonidina, el veralipride, la metoclopramida, la bromocriptina, la naloxona, los análogos del GnRH, la dopamina y afines, los andrógenos y los gestágenos, y la tibolona entre otros. Sin embargo, antes de iniciar la descripción de los distintos tipos de tratamientos no hormonales diremos que el tratamiento más efectivo es sin lugar a dudas el tratamiento hormonal con una eficacia que supera al $90 \%$ y que las alternativas estarían indicadas en mujeres que tienen contraindicados los estrógenos o que rechazan el tratamiento hormonal substitutivo.

Entre los tratamientos no hormonales de los síntomas vasomotores que se han citado anteriormente y se han mostrado efectivos en mayor o menor grado se incluyen la alfametildopa, la clonidina, la veraliprida, el proxibarbal y la beta-alanina. A continuación describimos sus características principales y en la tabla 4 se recogen las pautas de administración de los mismos.

\section{Tabla 3}

\section{COMPUESTOS NO ESTROGENICOS EN EL TRATAMIENTO DE LOS SOFOCOS}

- Sedantes y tranquilizantes

- Hormonas esteroideas no estrogénicas

- Progestágenos

- Andrógenos

- Esteroides sintéticos (Tibolona)

- Antiinflamatorios no esteroideos (AINE)

- Alfa - adrenérgicos

- B - bloqueantes

- Antidopaminérgicos

- Agonistas de los receptores de opiáceos

- Inhibidores de la aminoácido-descarboxilasa

- Antiestrógenos no esteroidales

- Alfametildopa: es un agonista alfa-2-adrenérgico que restablece el desequilibrio noradrenérgico. Su eficacia se sitúa en alrededor del $20 \%$.

- Clonidina: es también al igual que el anterior un agonista alfa-2-adrenérgico que restablece asimismo el desequilibrio noradrenérgico. Tiene también acción periférica ejercida mediante receptores alfa a nivel del sistema vascular. Su eficacia está alrededor del $40 \%$. No sólo disminuye la frecuencia e intensidad de los sofocos (22-27), sino que también disminuye los niveles de LH (23) así como la amplitud y frecuencia de los pulsos de la misma (24). Si bien tan sólo un autor (26) no ha detectado modificaciones importantes de los sofocos tras administrar clonidina, sus efectos secundarios no la hacen aconsejable en pacientes normotensas (25). La lofexidina es, al igual que la clonidina, otro alfaadrenérgico que puede emplearse en el tratamiento de los 
sofocos, sin embargo presenta en exceso hipotensión arterial (27).

- Veraliprida: es probablemente el compuesto no hormonal más utilizado y múltiples estudios han demostrado su utilidad (28-30). Es una benzamina antidopaminérgica central que aumenta también la actividad opiácea (29). Su eficacia varía entre el 60-90\% según los autores y produce no sólo una disminución del número y la intensidad de los bochornos, sino también una mejoría de los trastornos neuropsíquicos asociados a la menopausia (irritabilidad, nerviosismo, estado depresivo, ansiedad, insomnio). Las pacientes permanecen asintomáticas incluso más allá de 3 meses después de cesar la administración del fármaco. Entre sus efectos secundarios se cuentan la hiperprolactinemia. La metoclopramida, la domperidona y el sulpiride son otros fármacos antidopaminérgicos con características farmacológicas parecidas a las del veralipride. Todos estos fármacos provocan descensos en la concentración de la LH.

- Proxibarbal: interfiere en el sistema serotoninérgico. Tiene una eficacia que oscila entre el 60 y $90 \%$ y actúa también sobre la ansiedad, la inestabilidad y el insomnio.

- Beta-alanina: es un aminoácido puro que actúa como vasodilatador periférico.

Tabla 2

DOSIFICACIONES DE LOS PRINCIPALES FARMACOS NO HORMONALES QUE HAN SIDO UTILIZADOS PARA EL TRATAMIENTO DE LOS SOFOCOS

\begin{tabular}{|l|l|c|}
\hline Principio activo & \multicolumn{1}{|c|}{ Nombre comercial } & $\begin{array}{c}\text { Dosis } \\
\text { (mg/día) }\end{array}$ \\
\hline Análogos de la & SHRH & \\
Bromocriptina & Parlodel (Syntex) & 0,4 \\
Clomifeno & Clomifen (Casen) & $2,5-5$ \\
Clonidina & Catapresan (Boehringer) & $0,15-0.30$ \\
Danazol & Danogar (Wintrop) & 100 \\
Metildopa & Aldomet (Aldo unión) & $250-750$ \\
Metoclopramida & Primperan (Delagrange) & $10-20$ \\
Naloxona & Naloxona (Abelló) & $0.4-0,8$ \\
Veralipride & Agreal (Delagrange) & 200 \\
\hline
\end{tabular}

Aparte de los anteriores, también se consideró en su tiempo cierto grupo de fármacos como el naproxeno, pero después de las expectativas provocadas por el estudio de Edgren (31) que refería un descenso del 50\% de los bochornos, no ha vuelto a ser considerado como un fármaco útil en este campo y actualmente se piensa que su acción no va más allá del efecto placebo. La bromocriptina, un fármaco con acciones contrarias al veralipride, también ha sido utilizado, así mientras el primero es un antidopaminérgico, el segundo refuerza la acción central de la dopamina. A pesar de esta diferencia, la bromocriptina ha demostrado cierta utilidad en diversos estudios (32-34).
También se ha estudiado a la naloxona, un antagonista de los opiáceos, ya que se sabe que las endorfinas están implicadas en la producción de los sofocos; la administración de endorfinas induce bochornos, mientras que la naloxona los hace desaparecer (35), pero el hecho de tener que inyectarla para administrarla, mientras que el veralipride es oral y con mejores resultados, ha hecho que no se le considere como una opción práctica en el tratamiento de los sofocos. También se creyó en su día en el empleo de los análogos de la LHRH por su acción antigonadotropa; habiendo sido usados por Casper y Yen (36) en el tratamiento de los sofocos, sin embargo, tanto el costo de estas terapias como los diversos efectos secundarios, entre ellos el incremento de bochornos y la pérdida de masa ósea hacen desaconsejable esta terapia. Finalmente, algunos autores (37) han comparado la utilidad de los B-bloqueantes (Sotalol) frente a las benzodiazepinas (Lorazepam) y han observado una mayor eficacia de los primeros $(62 \%$ de descenso en la aparición de bochornos) frente a los segundos (37\%). Sin embargo los $\beta$-bloqueantes no tienen ninguna acción frente a síntomas de la menopausia tales como la ansiedad o el insomnio.

Otros fármacos que han demostrado su utilidad en el tratamiento de los bochornos son los gestágenos (38-39), los andrógenos (40-42) y la tibolona (43-44), sin embargo por su carácter hormonal no los incluimos en esta revisión.

Existen además otras medidas fisicodietéticas que pueden contribuir a disminuir la frecuencia e intensidad de los síntomas vasomotores. Así recientemente Ivarsson (45) muestra como la actividad física reduce la sintomatología, y aunque reconoce que puede haber un sesgo de selección en su estudio, sugiere que el aumento de endorfinas que produce el ejercicio podría explicar este efecto beneficioso, a través de su acción sobre los neurotransmisores que regulan el centro termorregulador.

En cuanto a la dieta, actualmente se habla mucho de los fitoestrógenos los cuales si bien están ampliamente distribuidos en el reino vegetal, los que más se conocen y se ingieren son básicamente los obtenidos de la soya. Es bien conocido que las culturas orientales y especialmente la japonesa ingieren altas cantidades de soya y esto podría ser la explicación de la baja incidencia de síntomas climatéricos en esas regiones. Los isoflavonoides o fitoestrógenos tienen una débil capacidad estrogénica, pero ingeridos a altas dosis pueden llegar a compensar la carencia de estrógenos. Así Adlercreutz (46) estudia la excreción urinaria de estrógenos y fitoestrógenos en la población japonesa y la compara con una población finlandesa, encontrando unas diferencias de hasta 100 veces mayor de fitoestrógenos en las japonesas, mientras que las concentraciones de estrona, estriol y estradiol son similares en ambas poblaciones.

Actualmente se están llevando a cabo numerosos estudios con los fitoestrógenos. Son compuestos difenólicos obtenidos de las plantas que tienen actividad estrogénica y antiestrogénica en humanos y animales. Además, tienen actividad antioxidante y pueden actuar protegiendo frente a la enfermedad cardiovascular y a los cánceres. 
Los isoflavonoides más conocidos que contiene la soya son genistein, daidzein y equol. Genistein tiene la mayor actividad como fitoestrógeno, pero equol tiene mayor actividad antioxidante. Sin embargo, para Mitchell (47) la actividad antioxidante de los fitoestrógenos sería escasa a las concentraciones que se alcanzan en el suero.

La soya y otros vegetales contienen importantes isoflavonoides que son biotransformados por la flora intestinal, absorbidos, reciclados a nivel enterohepático y alcanzan en la circulación concentraciones que superan en mucho a los estrógenos endógenos. Estos fitoestrógenos tienen importantes acciones hormonales y no hormona- les que pueden explicar sus efectos biológicos (48). Así pues, nos encontramos ante una alternativa no farmacológica que parecería ser una buena opción para las pacientes con sintomatología climatérica.

Podríamos concluir, señalando que existen alternativas a la terapia hormonal de reemplazo, que aisladamente o en combinación, permiten tratar los bochornos que presentan las pacientes que tienen contraindicación o no desean usar hormonas durante el climaterio. La aplicación de estas alternativas cambiará significativamente la calidad de vida de estas mujeres.

\section{BIBLIOGRAFIA}

1. Ledesert B; Ringa V; Brean G. Menopause and perceived health status among the women of the French GAZEL cohort. Maturitas 1994; 20 (2-3): 113-20.

2. Buvat J, Buvat-Herbaut M. Physiopatologie des bouffées de chaleur de la ménopause. Rev Fr Gynecol Obstet. 1981; 76: 661-666.

3. Fink PJ. Psychiatric myths of the menopause. In: The menopause comprehensive management. Eskin BA ed. Masson Publishing. New York (USA). 1980; (111-118).

4. Rebar RW, Spitzer 1B. The physiology and measurement of hot flushes. Am J Obstet Gynecol 1987; 1565: 1284-1288.

5. Freedman RR. Laboratory and ambulatory monitoring of menopausal hot flashes. Psychophysiology. 1989; 26: 573-9.

6. Haas S., Schiif I. Síntomas de deficiencia de estrógenos. En: Studd JWW, Whitehead MI. Menopausia. Ancora SA. Barcelona. 1990; 17-26.

7. Rees MC., Barlow DH. Absence of sustained reflex vasoconstriction in women with menopausal flushes. Human Reprod. 1988; 3: 823-5.

8. Bates GW. On the nature of the hot flash. Clin Obstet Gynecol N A. 1981; 24: 231-242.

9. Parrilla JJ, Abad L. La clínica del climaterio. En: Embarazo en la Adolescencia y Menopausia y Climaterio. Libro de ponencias. 19 Congreso Nacional de Ginecología. Oviedo. Octubre 1987; (123165).

10. Voda AM. Climateric hot flash. Maturitas. 1981; 3: 73-90.

11. Blümel JE, Roncagliolo ME, Gramegna G, Tacla X, Sepulveda H, Brandt A. Prevalencia de síntomas psíquicos y vasomotores en diferentes períodos del climaterio. Rev Chil Obstet Ginecol 1997; 62: 412-18.

12. Kronenberg F, Cote LJ, Linkie DM, Dyrenfurth I, Downey JA. Menopausal hot-flashes: thermoregulatory cardiovascular and circulating catecholamines and LH changes. Maturitas 1984; 6: 31-43.

13. Frishman GN. The hot flash: pathophysiology and treatment. IR Med, May 1995; 78(5): 132-4.

14. Freedman RR. Biochemical, metabolic, and vascular mechanisms in menopausal hot flashes. Fertil Steril 1998; 70: 332-7.

15. Tepper R., Pardo J., Ovadia J., Beyth Y. Menopausal hot flushes, plasma calcitonin and beta-endorphin. Gynecol Obstet Invest 1992; 33: 98-101.

16. Tepper R, Nerio A, Kaufman H, Schoenfeld A, Ovadia J. Menopausal hot flashes and plasma beta-endorphins. Obstet Gynecol 1987; 70: 150-2.

17. Simpkins JW. Similarities between morphin withdrawal in the rat and the menopausal hot flush. Maturitas, 1980; 2: 101-7.

18. D'Amico JF., Greendate GA., Lu IK., Judd HL. Induction of hypothalamic opioid activity with transdermal estradiol administration in postmenopausal women. Fertil Steril 1991; 55: 754-8.

19. Cignarelli M., Cicinelli E., Corso M., Cospite MR., Garruti G., Taforo E., Giorgino R., Schonauer S. Biophysical and endocrine metabolic changes during menopausal hot flashes: increase in plasma free fatty acid and norepinephrine levels. Gynecol Obstet Invest 1989; 27: 34-37.

20. Drapier-Faure E. Ménopause et son traitement- Editions TechniquesEncycl. Méd. Chir. Gynecologie 38-A-10, 1992.
21. Ferrer Barriendos J. Repercusiones clínicas a corto plazo: síndromes vasomotor y psiconeurobiológico. En: Cuadernos de Medicina Reproductiva. Climaterio y menopausia. ISSN: 1335-0970. 1999.

22. Laufer LR., Erlik Y., Meldrum DR., Judd HL. Effect of clonidine on hot flashes in postmenopausal women. Obstet Gynecol. 1982; 60: 583-586.

23. Baranowska $B$. The effect of clonidine on pituitary hormone secretion in physiological and pathological states. J Cardiovasc Pharm. 1987; 10: $235-239$.

24. Ginsburg J., O'Reilly B. Effect of oral clonidine on the vascular effects of stressful mental arithmetic in menopausal women. J Cardiovasc Pharm. 1987; 10: 138-141.

25. Wren BG., Brown LB. A double-blind trial with clonidine and a placebo to treat hot flushes. Med J Austr. 1986; 144: 369-379.

26. Lindsay R., Hart DM. Failure of response of menopausal vasomotor symptoms with clonidine. Maturitas. 1978; 1: 21-25.

27. Jones KP., Ravnikar V., Schiff I. A preliminary evaluation of the effect of lofexidine on vasomotor flushes in postmenopausal women. Maturitas. 1985; 7: 135-139.

28. Boukobza G. Efficacité et tolerance du véralipride dans le traitement des bouffées de chaleur de la ménopause. Etude multicentrique. Rev Fr Gynécol Obstét. 1986; 81: 413-417.

29. David A., Don R., Tajchner G., Weissglas L. Veralipride: alternative antidopaminergic treatment for menopausal symptoms. Am J Obstet Gynecol. 1988; 158: 1107-1117.

30. Wesel S., Bourguignon RP, Bosuma WB. Veralipride versus conjugated estrogens: a double-blind study in the management of menopausal hot flushes. Current Med Res Op. 1984; 8: 696-700.

31. Edgren RA., Morton CJ. Naproxen sodium for OB/GYN use, with special reference to pain states: a review. International Journal of fertility. 1986; 31: 135-142.

32. Kibanski A., Groenspan SL. Increase in bone mass after treatment of hyperprolactinemic amenorrhea. N Engl J Med 1986; 315: 542-544.

33. Melis GB., Paoletti AM., Mais V. Inhibiting effect of the dopamine agonist bromocriptine on the postcastrational gonadotropin rise in women. Clin Endocrinol (Oxf), 1981; 31: 530-535.

34. Zichella L., Falaschi P., Fioretti P et al. Effects of different dopamine agoniøsts and antagonists in menopausal hot flushes. Maturitas. 1986; 8: 229-239.

35. De Fanzio J., Verheugen C., Chetkowski R., Nass T., Judd HL et al. The effects of Naloxone on hot flashes and gonadotropin secretion in postmenopausal women. J Clin Endocrinol Metab. 1984; 58: 578-581.

36. Casper RF., Yen SSC. Menopausal hot flushes: effect of pituitary gonadotropin desensitization by a potent LHRH agonist. J Clin Endocrinol Metab. 1981; 53: 1056-1059.

37. Dallera F., Buzzi S., Jezzi BG., Gendarini A. Sotalo nei disturbi menopausali. Clínica Terapéutica. 1986; 119: 465-469.

38. Gambrell RD. Clinical use of progestins in the menopausal pacient: dosage and duration. J Reprod Med. 1982; 27: 531-538.

39. Lobo RA., McCormick W., Singer F., Roy S. Depomedroxyprogesterone acetate compared with conjugated estrogens for the treatment of postmenopausal women. J Am Col Obstet Gynecol. 1984; 63: 105. 


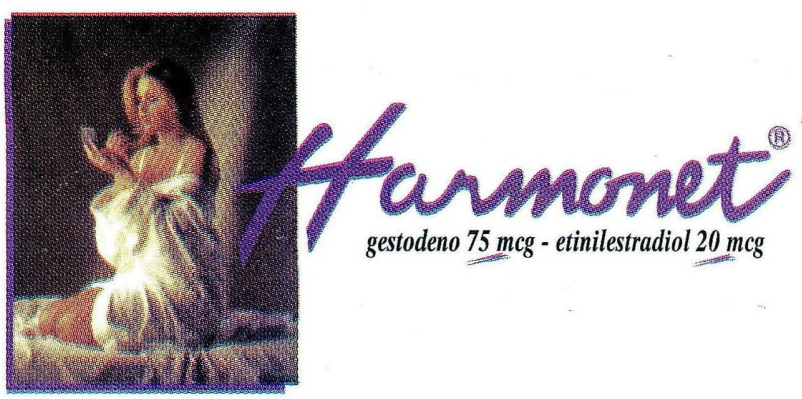

FORMULA

Cada gragea contiene: Gestodeno $75 \mathrm{mcg}$, Etinilestradiol $20 \mathrm{mcg}$, Lactosa $37,155 \mathrm{mg}$, azúcar 19,66 mg almidón $\mathrm{mg}$, estearato de magnesio $550 \mathrm{mcg}$, povidona K-90 $171 \mathrm{mcg}$, cera E 50 mog.

\section{INDICACIONES}

Harmonet está indicado para la prevención del embarazo en mujeres que deciden utilizar este método.

\section{POSOLOGIA Y FORMA DE ADMINISTRACION}

Para lograr una eficacia anticonceptiva óptima, HARMONET deberá ingerirse diariamente según las indicaciones y con intervalos diarios que no excedan las 24 horas. Debe instruirse a la paciente para que tome las grageas todos los dias a la misma hora, preferiblemente con la comida de la noche 0 bien antes de acostarse.

\section{CONTRAINDICACIONES}

Harmonet está contraindicado en las mujeres que presenten: Antedentes o diagnóstico de trastornos trombóticos arteriales o venosos, trastornos embólicos o situaciones que pudieran predisponer a la paciente (por ejemplo, defectos de la coagulación, valvulopatías cardiacas, fibrilación uricular); transtornos agudos o crónicos de la función hepática (incluyéndose entre éstos el sindrome de DubinJohnson 0 al sindrome de Rotor), antecedentes o diagnostico del tumores hepáticos antecedentes de ictericia diopatica o de prurito importante durante el embarazo; antecedentes o diagnostico conocido o sospechado de neoplasias hormonodependientes ( por ejemplo, cancer de mama o de endometrio); trastornos del metabolismo lipidico; anemia falciforme; diabetes mellitus con alteraciones vasculares; antecedentes de herpes gestacional;
otosclerosis que se agravan durante el embarazo; sangrado vaginal no diagnósticado; embarazo conocido o sospechado; hipersensibilidad a cualquiera de los componentes de Harmonet.

\section{EFECTOS SECUNDARIOS}

Los siguientes efectos secundarios se han registrado en un total de 1338 pacientes que recibieron Harmonet durante el curso de tres estudios clínicos:

Generales Incidencia >3\%: dolor abdominal, dolor de espalda, dolor en los genitales, dolor pélvico. Incidencia < que 1\%: distensión del abdomen, abcesos, reacciones alérgicas, astenia, fiebre, sindrome gripal, pesadez en las extremidades, infecciones, maleśtar, moniliasis, artritis reumatoidea. Sistema Cardiovascular Incidencia $>3 \%$ : migraña. Incidencia de $1 \%-3 \%$ : venas varicosas. Incidencia < $1 \%$ : dolor al pecho trombosis de las venas profundas sofocos, hipertensión, palpitaciones, taquicardia, tromboembolia, tromboflebitis, vasodilatación, y otros transtornos cardiovasculares. Sistema Digestivo Incidencia $<3 \%$ : Náuseas. Incidencia 1\%-3\%: Vómito. Incidencia <1\%: colecistis, diarrea, flatulencia, transtornos vesiculares,gastritis, gastroenteritis, transtornos gastrointestinales, hepatopatía, aumento del apetito estomatitis. Sistema Metabólico Incidencia < $<\%$ edema aumento o pérdida del peso. Sistema Nervioso Incidencia $>3 \%$ : Cefaleas, nerviosismo. Incidencia $1 \%-3 \%$ : Depresión, mareos, alteraciones en la libido. Incidencia < $1 \%$ : amnesia, ataxia, hostilidad, parestesia, transtornos en el sueño, somnolencia, sudoración excesiva. Sistema Respiratorio incidencia <1\%: bronquitis, faringitis, rinitis, sinusitis. Dermatológicos. Incidencia >3\%: Acné. Indicidencia < 1\%: alopecía, cloasma, eczema, prurito, eritema, otros trastornos dermatológicos. Organos de los sentidos. Incidencia <1\%: visión anormal, tinnitus, sordera total transitoria. Sistema Urogenital. Incidencia $>3 \%$ : amenorrea, sangrando intermestrual, goteo, tensión mamaria. Incidencia < \% : cistitis, d ismenorrea, disuria, dolor en los genitales, galactorrea, leucorrea, nefritis, quistes ováricos, cálculos renales infecciones del trato urinario, sequedad vaginal, moniliasis vaginal, vaginitis, transtornos vulvovaginales. Por otra parte, los siguientes efectos secundarios se han registrado en pacientes que recibian anticonceptivos orales y se consideraron relacionados con la droga administrada:Náuseas, Vómitos, síntomas gastrointestinales, (Tales como cólicos abdominales e inflamación), sangrado intermenstrual, goteo, alteraciones en el flujo menstrual, amenorrea, infertilidad temporaria con posterioridad a la descontinuación del tratamiento, edema, melasma que puede persistir, aumento o disminución de peso, alteraciones en la erosión y secreción cervical, disminución en el volumen de leche en el postparto inmediato, ictericia colestática, migraña, eritema (alérgico), depresión mental, menor tolerancia a los carbohidratos, candidiasis vaginal, acentuación de la curvatura de la córnea, intolerancia a los lentes de contacto, cambio en las mamas: tensión, aumento de tamaño, secreción. Existen evidencias de una posible relación entre los siguientes efectos secundarios y la utilización de anticonceptivos orales, aunque aún debe confirmarse esta información: Trombosis mesentérica, Trombosis retiniana. Los siguientes efectos secundarios se han registrado en pacientes que recibían anticonceptivos orales, pero su asociación no ha sido aún confirmada ni rechazada: Anomalias congénitas, Síndrome premestrual, cataratas, neuritis óptica, cambios en el apetito, sindrome similar a la cisitits, cefaleas, nenviosismo mareos, hirsutismo pérdida del cabello, eritema multiforme, eritema nodoso, erupción hemorrágica, váginitis, disminución de la función renal, sindrome urémico hemolítico sindrome de BuddChiari, acné, cambios en la libido colitis, enfermedad cerebrovascular, con prolapso de la válvula mitral, sindrome similares a lupus.

Para mayor información, véanse las advertencias y precauciones.

\section{SOBREDOSIS}

La sobredosis puede causar náuseas o vómitos; en las mujeres puede producirse sangrado por supresión. En niños, no se han registrado efectos graves después de la utilización de altas dosis de anticonceptivos orales.

\section{PRESENTACION}

Envase con 21 grageas. Registro INVIMA 006606
40. Foster GV., Zacur HA., Rock JA. Hot flashes in postmenopausal women ameliorated by danazol. Fertil Steril. 1985; 43: 401-404.

41. Young RL., Kumar NS., Goldzieher JW. Management of menopause when estrogen cannot be used. Drugs. 1990; 40: 220-230.

42. NAMS roundtable. Physiologic androgen replacement in menopausal women. Menopause Management. 1998; 7: 26-28.

43. Kicovik PM., Cortés-Prieto J., Luisi M., Milojevic S., Franchi F. Placebo controlled cross-over study of effects of Org OD 14 in menopausal women. Reproducción, 1982; 6: 81-91.

44. Volpe A., Facchinetti F., Grasso A., Petraglia F., Campanini D, et al Benefits and risks of different hormonal replacement therapies in postmenopausal women. Maturitas. 1986; 8: 327-334.

45. lvarsson T., Spetz AC., Hammar M. Physical exercise and vasomotor symptoms in postmenopausal women. Maturitas, Jun 3 1998; 29 (2): 139-46.

46. Adlercreutz H. Dietary phyto-oestrogens and the menopause in Japan. Lancet May 16, 1992; 1233.

47. Mitchell JH., Gardner PT., McPhail DB., Morrice PC., Collins AR. Duthie GG. Antioxidant efficacy of phytoestrogens in chemical and biological model systems. Arch Biochem Biophys 1998; Dec 1; 360(1): 142-8.

48. Setchell KD. Phytoestrogens: the biochemistry, physiology, and implications for human health of soy isoflavones. Am J Clin Nutr 1998; Dec; 68 (6 suppl): 1333S-1346S.

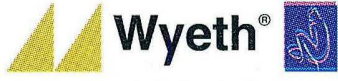

Lider mundial en el cuidado de la salud femenin 